Journal for ImmunoTherapy of Cancer

\section{Phase II study of durvalumab plus tremelimumab as therapy for patients with previously treated anti-PD-1/PD-L1 resistant stage IV squamous cell lung cancer (Lung-MAP substudy S1400F, NCT03373760)}

To cite: Leighl NB, Redman MW, Rizvi N, et al. Phase II study of durvalumab plus tremelimumab as therapy for patients with previously treated anti-PD-1/ PD-L1 resistant stage IV squamous cell lung cancer (Lung-MAP substudy S1400F, NCT03373760). Journal for ImmunoTherapy of Cancer 2021;9:e002973. doi:10.1136/ jitc-2021-002973

\section{- Additional online} supplemental material is published online only. To view, please visit the journal online (http://dx.doi.org/10.1136/jitc2021-002973).

NBL, MWR and NR contributed equally.

Accepted 19 July 2021

Check for updates

(c) Author(s) (or their employer(s)) 2021. Re-use permitted under CC BY-NC. No commercial re-use. See rights and permissions. Published by BMJ.

For numbered affiliations see end of article.

Correspondence to Dr Natasha B Leighl; natasha.leigh|@uhn.ca

\section{ABSTRACT}

Introduction S1400F is a non-match substudy of Lung Cancer Master Protocol (Lung-MAP) evaluating the immunotherapy combination of durvalumab and tremelimumab to overcome resistance to antiprogrammed death ligand 1 (PD-(L)1) therapy in patients with advanced squamous lung carcinoma (sq non-smallcell lung cancer (NSCLC)).

Methods Patients with previously treated sqNSCLC with disease progression after anti-PD-(L)1 monotherapy, who did not qualify for any active molecularly targeted Lung-MAP substudies, were eligible. Patients received tremelimumab $75 \mathrm{mg}$ plus durvalumab $1500 \mathrm{mg}$ once every 28 days for four cycles then durvalumab alone every 28 days until disease progression. The primary endpoint was the objective response rate (RECIST V.1.1). Primary and acquired resistance cohorts, defined as disease progression within 24 weeks versus $\geq 24$ weeks of starting prior anti-PD-(L)1 therapy, were analyzed separately and an interim analysis for futility was planned after 20 patients in each cohort were evaluable for response. Results A total of 58 eligible patients received drug, 28 with primary resistance and 30 with acquired resistance to anti-PD(L)1 monotherapy. Grade $\geq 3$ adverse events at least possibly related to treatment were seen in $20(34 \%)$ patients. The response rate in the primary resistance cohort was $7 \%(95 \%$ $\mathrm{Cl} 0 \%$ to $17 \%$ ), with one complete and one partial response. No responses were seen in the acquired resistance cohort. In the primary and resistance cohorts the median progression-free survival was 2.0 months ( $95 \% \mathrm{Cl} 1.6$ to 3.0 ) and 2.1 months (95\% Cl 1.6 to 3.2), respectively, and overall survival was 7.7 months $(95 \% \mathrm{Cl} 4.0$ to 12.0$)$ and 7.6 months $(95 \% \mathrm{Cl} 5.3$ to $10.2)$, respectively.

Conclusion Durvalumab plus tremelimumab had minimal activity in patients with advanced sqNSCLC progressing on prior anti-PD-1 therapy.
Trial registration number NCT03373760.

\section{INTRODUCTION}

The Lung Cancer Master Protocol (Lung-MAP) was designed to employ tumor screening with broad-based next generation sequencing (NGS) in order to simultaneously evaluate multiple novel targeted therapies in patients with advanced, previously treated squamous lung carcinoma (sq nonsmall-cell lung cancer (NSCLC)). ${ }^{1}{ }^{2}$ This platform facilitates screening large numbers of patients to identify rare, actionable mutations or other unique molecular features and then matching the patient to a biomarkerdriven Lung-MAP substudy. Those without a biomarker match, or otherwise not qualified for a targeted substudy, could be enrolled into a 'non-match' substudy. After Lung-MAP was developed and began accrual, the standard of care for platinum-treated advanced sqNSCLC patients evolved from docetaxel chemotherapy to Programmed cell death protein 1 (PD-1)/programmed death -ligand 1 (PD-L1)-directed checkpoint inhibitor therapy. With response rates in the range of $20 \%$, a significant number of patients do not derive long-term benefit from secondline therapy with single agent PD-1 or PD-L1 inhibitors, and the majority of those with initial response subsequently progress. ${ }^{3-5} \mathrm{~A}$ similar pattern has emerged as PD-1 or PD-L1 
inhibitors have moved to the first-line setting, with or without chemotherapy. Greater understanding of the scientific basis underlying primary and acquired resistance to PD-1 checkpoint inhibitors, and options to overcome this resistance, are urgently needed.

Limited data are available on mechanisms of resistance to PD-1 checkpoint inhibitor therapy in lung cancer. There is growing interest in genomic correlates of PD-1 checkpoint inhibitor response, including increased tumor mutational burden (TMB) and DNA damage response and repair (DDR) gene alterations. ${ }^{6-8}$ Failure of engagement of tumor-infiltrating $\mathrm{T}$ cells by tumor antigens, an immunosuppressive tumor microenvironment, impaired T-cell effector function, and signaling via alternate immune inhibitory checkpoints are all potential mechanisms of resistance. Cytotoxic T-lymphocyteassociated protein 4 (CTLA-4) and PD-1 checkpoints are both negative regulators of $\mathrm{T}$ cell activation and function, and use distinct mechanisms to block $\mathrm{T}$ cell activity. CTLA-4 decreases signaling via CD28, leading to impaired T-cell activation, while PD-1 inhibition results in $\mathrm{T}$ cell effector dysfunction. Preclinical and clinical studies demonstrate synergy when CTLA-4 and PD-1 axis inhibitors are combined, which has led to superior outcomes with combination immunotherapy in melanoma and renal cell carcinoma. ${ }^{9-12}$ The combination of CTLA- 4 and PD-1 axis inhibitors has also emerged as a standard option in the treatment of advanced pleural mesothelioma and one of several first-line options for patients with advanced NSCLC. ${ }^{13} 14$

We explored adding tremelimumab, a fully humanized $\operatorname{IgG}_{2}$ monoclonal antibody that binds CTLA- 4 on activated $\mathrm{T}$ cells, to ongoing PD-L1 inhibition with durvalumab, a human immunoglobulin G1 kappa (IgG1א) monoclonal antibody, in patients with advanced pre-treated sqNSCLC and primary or acquired resistance to PD-(L) 1 inhibitors. We also explored the relationship of PD-L1 tumor expression with patient outcomes, and post-hoc exploration of outcomes by TMB and somatic mutations in DDR genes, potential correlates of checkpoint inhibitor response.

\section{MATERIALS AND METHODS \\ Study design}

This multicenter, open-label, phase II trial and substudy of Lung-MAP (S1400), was conducted through the National Clinical Trials Network and led by the SWOG Cancer Research Network as described previously. ${ }^{12}$ Patients were stratified into two cohorts based on prior response to checkpoint inhibitor monotherapy. Primary PD-(L) 1 resistance was defined as disease progression within 24 weeks of initiation of single agent anti-PD-1/PD-L1 therapy. Acquired PD-(L) 1 resistance was defined as 24 weeks or more of disease control (complete response, partial response, or stable disease) after initiation of single agent anti-PD-1/PD-L1 therapy that had subsequently progressed after 24 weeks. The trial assessed outcomes of durvalumab plus tremelimumab-treated patients with primary vs acquired resistance to anti-PD $(\mathrm{L}) 1$ therapy.

\section{Eligibility}

Patients must have been eligible for the Lung-MAP (S1400) screening study and not eligible for any of the actively accruing biomarker-driven sub-studies. ${ }^{2}{ }^{15-18}$ Other eligibility criteria included: histologically confirmed stage IV or recurrent sqNSCLC with measurable disease per RECIST V.1.1, prior platinum-based chemotherapy, and progression during or after anti-PD-1 or anti-PD-L1 antibody monotherapy as their most recent line of treatment, no prior treatment with CTLA-4 inhibitors, no immunosuppressive medication nor attenuated vaccinations within 28 days, and no systemic corticosteroids within 24 hours prior to registration. Patients with a history of organ transplant requiring immunosuppressive therapy were excluded. Patients could not have active, known, or suspected tuberculosis, HIV, AIDS, hepatitis B, hepatitis C, or autoimmune or inflammatory disease within 3 years. In addition, patients could not have experienced grade 3 or greater immune-mediated toxicity, except asymptomatic rash, nor any toxicity that led to permanent discontinuation of prior anti-PD-1/PD-L1 therapy. Patients were stratified into two cohorts for analysis purposes, primary anti-PD-1/PD-L1 resistance and acquired anti-PD-1/ PD-L1 resistance.

Tremelimumab $75 \mathrm{mg}$ was administered intravenously over 1 hour, followed by durvalumab $1500 \mathrm{mg}$ intravenous administration over 1 hour on day 1 of 28-day cycles for the first four cycles. Starting at cycle 5, only durvalumab $1500 \mathrm{mg}$ was administered on day 1 of each cycle as maintenance until disease progression or unacceptable toxicity. Disease assessment occurred every two cycles, and treatment was continued until disease progression or unacceptable toxicity. Only dose interruptions or discontinuations were allowed to manage toxicity and were discussed with the study chairs as specified in the protocol.

\section{Biomarker screening and analysis}

All Lung-MAP (S1400) patients had tumor tissue biomarker screening by NGS by Foundation Medicine, as previously described. PD-L1 immunohistochemistry (IHC) staining and scoring was performed by Clarient, using the technically validated IHC-based SP263 assay developed by AstraZeneca for PD-L1 determination in partnership with Ventana Medical Systems, a College of American Pathologists (CAP)-accredited/Clinical Laboratory Improvement Amendments-certified laboratory (Tucson, AZ).

\section{Statistical considerations}

The primary objective was to evaluate the objective response rate (ORR; confirmed and unconfirmed, complete and partial) by RECIST version 1.1 in each cohort, the primary PD-(L) 1 resistant cohort, and the acquired PD-(L)1 resistant cohort. The sample size for 
each cohort was based on a design with $82 \%$ power to rule out an ORR of $15 \%$ at the $5 \%$ level if the true rate was $35 \%$. The accrual goal for each cohort was 66 patients to achieve 60 evaluable patients per cohort. The design included two interim analyses for each cohort separately at 20 and 40 patients evaluable for response, and the cohorts would continue accrual independently; at least one response was needed to accrue past the first interim analysis, and at least four responses were needed to accrue past the second interim analysis. If a study cohort reached full accrual, 6 or more responses were needed to rule out a $15 \%$ ORR. Other key secondary objectives to be analyzed by cohort included overall survival (OS), progression-free survival (PFS), and the relationship of PD-L1 expression status to response and PFS. Post hoc exploratory analysis of PFS by TMB and somatic mutations in DNA DDR genes, potential correlates of checkpoint inhibitor response and treatment outcomes, was also performed. ${ }^{78}$

\section{RESULTS}

\section{Patient characteristics and treatment}

Between October 22017 and March 24 2020, 1388 patients were genomically screened in Lung-MAP using NGS; 211 patients $(15 \%$ of those screened on S1400 while S1400F was actively accruing) were assigned to S1400F and 67 patients were enrolled. Seven patients were ineligible for study; six did not receive anti-PD-L1 monotherapy as their most recent line of treatment and one had inadequate documentation of measurable disease. Another two patients were ineligible for analysis, as one expired prior to receiving any treatment and one withdrew consent prior to treatment. In all, 58 eligible patients received protocol therapy, 28 in the primary resistance cohort and 30 in the acquired resistance cohort (online supplemental figure 1). Baseline characteristics were similar in the two cohorts and are described in table 1. Genomic alterations in tumor tissue are listed in online supplemental table 1 . The acquired and the primary resistance cohorts were permanently closed to accrual by the data and safety monitoring committee on November 62019 due to futility and on March 242020 due to poor accrual, respectively. Of note, the primary resistance cohort met the criterion to continue accrual past the first interim analysis.

\section{Efficacy}

There was one confirmed complete and one partial response, both in the primary resistance cohort for an ORR of $7 \%(95 \%$ CI $0 \%$ to $17 \%$ ); there were no responses in the acquired resistance cohort (table 2). The median duration of response for the two responding patients in the primary resistance cohort was 8.5 months and 5.9 months, respectively. The patient with partial response had a tumor PD-L1 score $<1 \%$, both responders had a tumor TMB of 15 mutations/ $\mathrm{Mb}$ and multiple somatic alterations including in TP53, PTEN, and PIK3 genes. Ten and 14 patients had stable disease in the primary and acquired resistance cohorts, respectively, with disease control rates at 12 weeks of $43 \%$ (95\% CI $25 \%$ to
Table 1 Patient demographics and characteristics

Primary PD-(L)1 Acquired PD-

resistance $\quad(\mathrm{L}) 1$ resistance

$(\mathrm{N}=\mathbf{2 8})$

$(\mathrm{N}=\mathbf{3 0})$

\begin{tabular}{|lcc|}
\hline Age median (range), years & $67.6(49.7-89.8)$ & $67.8(46.6-84.0)$ \\
\hline Male & $18(64)$ & $18(60)$ \\
\hline Race & & \\
\hline White & $24(86)$ & $26(87)$ \\
\hline Black & $3(11)$ & $3(10)$ \\
\hline Native American & $1(3)$ & $0(0)$ \\
\hline Not reported & $0(0)$ & $1(3)$ \\
\hline $\begin{array}{l}\text { Hispanic ethnicity } \\
\text { No of prior lines of therapy for stage IV disease }\end{array}$ & $3(10)$ \\
\hline$<2$ & $9(32)$ & $12(40)$ \\
\hline 22 (max 4) & $19(68)$ & $18(60)$ \\
$\begin{array}{l}\text { Median (range) PFS on } \\
\text { prior anti-PD-(L)1 therapy, } \\
\text { months }\end{array}$ & $3.0(1.4-5.5)$ & $10.0(5.6-30.4)$ \\
\hline
\end{tabular}

\begin{tabular}{|lcr|}
\multicolumn{3}{l}{ Best response to prior anti-PD-(L)1 therapy } \\
\hline Complete response $^{*}$ & $0(0)$ & $3(10)$ \\
\hline Partial response $^{*}$ & $2(7)$ & $7(23)$ \\
\hline Stable disease & $11(39)$ & $20(67)$ \\
Progressive disease & $15(54)$ & $0(0)$
\end{tabular}

Performance status

\begin{tabular}{lcc}
\hline & $7(25)$ & $10(33)$ \\
1 & $21(75)$ & $20(67)$ \\
Smoking status & \\
$\quad$ Current smoker & $10(36)$ & $10(33)$ \\
Former smoker & $17(61)$ & $19(63)$ \\
Never smoker & $1(4)$ & $1(3)$ \\
Weight loss $\geq 10 \%$ & $2(8)$ & $2(7)$ \\
PD-L1 expression† (TPS (\%)) & & $3(10)$ \\
$<1 \%$ & $10(36)$ & $9(30)$ \\
$1 \%-49 \%$ & $5(18)$ & $2(7)$ \\
$50 \%$ & $5(18)$ & $16(53)$ \\
\hline Unknown & $8(28)$ & $11(37)$ \\
Tumor mutational burden & & $17(57)$ \\
$<10$ mt/Mb & $8(28)$ & $2(6)$ \\
\hline 10 mt/Mb & $17(61)$ & \\
\hline Not evaluable & $3(11)$ & \\
\hline
\end{tabular}

Values are shown as $n(\%)$ unless otherwise stated.

*Includes confirmed and unconfirmed responses per investigator assessment.

†PD-L1 expression was assessed by immunohistochemistry on tumor samples using 22C3 pharmDx assay (Agilent Technologies, Santa Clara, California, USA).

PD-L1, programmed death ligand 1; TPS, Tumor Proportion Score.

$61 \%$ ) and $40 \%$ (95\% CI 23\% to 58\%). Figure 1 depicts the waterfall plot for individual responses color coded by cohort.

OS and investigator-assessed PFS (IA-PFS) were similar in both cohorts (figure 2). In those with primary resistance, the median IA-PFS was 2.0 months (95\% CI 1.6 to 3.0) and 
Table 2 Patient tumor responses

\begin{tabular}{lccc}
\hline & $\begin{array}{l}\text { Total } \\
(\mathbf{N}=58)\end{array}$ & $\begin{array}{l}\text { Primary PD-(L)1 Resistance } \\
(\mathbf{N = 2 8})\end{array}$ & $\begin{array}{c}\text { Acquired PD-(L)1 } \\
\text { Resistance (N=30) }\end{array}$ \\
\hline Complete response & $1(2)^{\star}$ & $1(4)$ & 0 \\
Partial response & $1(2)^{\star}$ & $1(4)$ & 0 \\
Stable disease/no response & $24(41)$ & $10(36)$ & $14(47)$ \\
Increasing disease & $27(47)$ & $12(43)$ & $1(50)$ \\
Symptomatic deterioration & $4(7)$ & $3(11)$ & 0 \\
Assessment inadequate & $1(2)$ & $1(4)$ & 0 \\
Objective response rate & $2(3(95 \% \mathrm{Cl} 0$ to 8$))$ & $2(7(95 \% \mathrm{Cl} 0$ to 17$))$ & $14(47(95 \% \mathrm{Cl} 29$ to 65$))$ \\
\hline Disease control rate & $26(45(95 \% \mathrm{Cl} 32$ to 58$))$ & $12(43(95 \% \mathrm{Cl} 25$ to 61$))$ &
\end{tabular}

Values are shown as $n$ (\%) unless otherwise stated.

*Includes confirmed and unconfirmed responses per investigator assessment.

PD-L1, programmed death ligand 1.

the median OS was 7.7 months (95\% CI 4.0 to 12.0 ). In the cohort with acquired resistance to anti-PD-(L)-1 inhibitor therapy, the median IA-PFS was 2.1 months $(95 \%$ CI 1.6 to 3.2 ) and the median OS was 7.6 months (95\% CI 5.3 to 10.2). Subgroup analysis did not identify any clinical or pathological characteristics associated with benefit, including age, performance status, sex, smoking status, PD-L1 tumor expression, nor tissue TMB, shown in figure 3. In post hoc analysis, patients with tumors harboring at least one or more alterations in DDR genes had similar IA-PFS and OS compared with patients with no tumor DDR genomic alterations (figure 3 , online supplemental figure 2 ).

\section{Safety}

Patients in both the primary and acquired resistance cohorts received a median of 3 cycles of durvalumab (range 1-14 in primary resistance, 1-16 in acquired resistance), and a median of 4 planned cycles of tremelimumab (range 1-4). There were two treatment-related deaths in the acquired resistance cohort (one pneumonitis, one death not otherwise specified possibly related to pneumonitis). The patient with fatal pneumonitis also experienced grade 4 dyspnea. Additionally, two patients experienced grade 4 adverse events due to lymphopenia



Figure 1 Waterfall plot of response to durvalumab plus tremelimumab. Of four patients with more than $30 \%$ reduction in tumor measurements, two had documented responses in the primary resistance cohort (1 partial and one complete response). One patient in the primary resistance cohort was found to have stable disease on independent radiology review. One patient in the acquired resistance cohort stopped treatment before disease progression (best response of stable disease) and started a new treatment prior to subsequent tumor measurements (included above). 
A

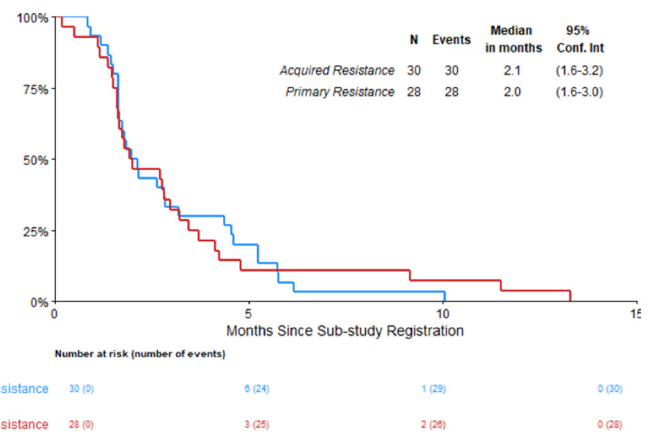

B

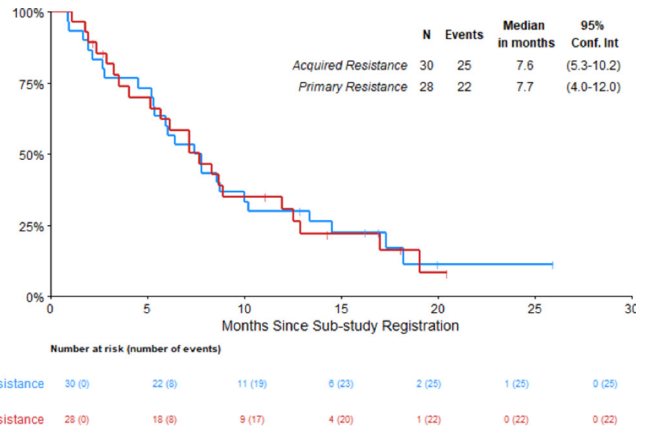

Figure 2 Kaplan-Meier curves of progression-free survival (PFS) and overall survival (OS) in the primary and acquired PD-(L)1 resistant cohorts. (A) PFS. (B) OS. PD-(L)1, programmed death ligand 1.

(1) and leukopenia (1). In the primary resistance cohort, one patient experienced treatment-related grade 4 thrombocytopenia. Overall, $33 \%$ of patients in the primary resistance and $36 \%$ of patients in the acquired resistance cohorts experienced grade three or higher treatment-related adverse events (table 3). Four patients in each cohort discontinued protocol therapy for toxicity. Further details of patient disposition are listed in online supplemental figure 1 .

\section{DISCUSSION}

In patients with advanced pretreated sqNSCLC and acquired resistance to anti-PD-1/PD-L1 monoclonal antibody therapy, no activity was seen with the addition of CTLA-4 inhibition to ongoing PD-1 axis inhibition with durvalumab plus tremelimumab. In patients with primary resistance, the ORR was $7 \%$. Both responders had higher
TMB (15 mutations/Mb) including alterations in TP53 and other genes, while one had tumor PD-L1 $<1 \%$ and the other had PD-L1 positive tumor expression. While this cohort was closed due to poor accrual, it is unlikely that a larger sample size would have yielded clinically different results. Toxicity was as expected for the combination and there were no characteristics associated with greater benefit from therapy, including PD-L1 tumor expression, tissue TMB, nor the presence of DDR mutations.

At the time this study was designed, second-line single agent anti-PD-(L) 1 therapy was a new standard for patients with advanced NSCLC, and upfront combinations of chemotherapy plus checkpoint inhibitors first-line had not yet been approved. Similarly, the use of combination nivolumab plus ipilimumab had not been approved in the first-line setting. The clinical benefit of adding CTLA-4 inhibition to PD-(L)1 checkpoint inhibition remains

\section{A}

Zubrod 0 vs. 1

Current vs. Former/Never Smoker

Male vs. Female

PD-L1 $>=1 \%$ vs. $<1 \%$

PD-L1 $>=50 \%$ vs. $<50 \%$

TMB >=10 vs. $<10$ mut/Mb

DDR Gene Mutation Yes vs. No
Age $65+$ vs. Less than 65

$\begin{array}{cccc}\text { Events/N } & \text { Events/N } & \text { HR }(\mathbf{9 5} \% \mathbf{C I}) & \text { P-value } \\ 39 / 39 & 19 / 19 & 1.06(0.61,1.85) & 0.83 \\ 17 / 17 & 41 / 41 & 1.07(0.61,1.90) & 0.81 \\ 20 / 20 & 38 / 38 & 1.13(0.65,1.95) & 0.67 \\ 36 / 36 & 22 / 22 & 0.76(0.44,1.31) & 0.32 \\ 21 / 21 & 13 / 13 & 0.99(0.48,2.04) & 0.98 \\ 7 / 7 & 27 / 27 & 1.27(0.54,3.02) & 0.59 \\ 34 / 34 & 19 / 19 & 0.62(0.34,1.15) & 0.13 \\ 16 / 16 & 42 / 42 & 1.16(0.64,2.07) & 0.63\end{array}$



B

$\begin{array}{lcccc} & \text { Events/N Events/N } & \text { HR }(95 \% \text { CI) } & \text { P-value } \\ \text { Age 65+ vs. Less than 65 } & 34 / 39 & 13 / 19 & 1.59(0.83,3.03) & 0.16 \\ \text { Zubrod 0 vs. 1 } & 14 / 17 & 33 / 41 & 0.95(0.51,1.80) & 0.88 \\ \text { Current vs. Former/Never Smoker } & 17 / 20 & 30 / 38 & 1.34(0.73,2.47) & 0.34 \\ \text { Male vs. Female } & 29 / 36 & 18 / 22 & 0.80(0.44,1.43) & 0.45 \\ \text { PD-L1 >=1\% vs. <1\% } & 17 / 21 & 9 / 13 & 1.63(0.72,3.72) & 0.24 \\ \text { PD-L1 >=50\% vs. <50\% } & 6 / 7 & 20 / 27 & 2.40(0.92,6.29) & 0.07 \\ \text { TMB >=10 vs. <10 mut/Mb } & 28 / 34 & 15 / 19 & 1.02(0.54,1.91) & 0.96 \\ \text { DDR Gene Mutation Yes vs. No } & 13 / 16 & 34 / 42 & 1.06(0.55,2.03) & 0.86\end{array}$

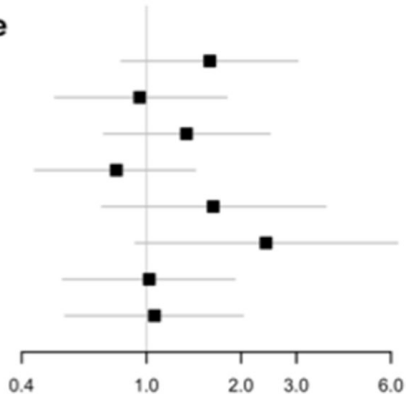

Figure 3 Forest plot comparisons of patient characteristics and progression-free survival (PFS) and overall survival (OS) in the full eligible population. (A) Forest plot for PFS. (B) Forest plot for OS. DDR, DNA damage response and repair; MB, megabase; mut, mutation; PD-L1, programmed death ligand 1; TMB, tumor mutational burden. 
Table 3 AEs attributable to treatment

\begin{tabular}{|c|c|c|c|c|c|c|}
\hline \multirow[b]{3}{*}{ AEs } & \multicolumn{3}{|c|}{ Primary PD-(L)1 Resistance (N=30) } & \multicolumn{3}{|c|}{ Acquired PD-(L)1 Resistance (N=28) } \\
\hline & \multicolumn{3}{|l|}{ Grade } & \multicolumn{3}{|l|}{ Grade } \\
\hline & 3 & 4 & 5 & 3 & 4 & 5 \\
\hline Atrial fibrillation & $1(3)$ & & & & & \\
\hline Atrial flutter & $1(3)$ & & & & & \\
\hline Chills & $1(3)$ & & & & & \\
\hline Confusion & & & & $1(4)$ & & \\
\hline Creatinine increased & & & & $1(4)$ & & \\
\hline Death NOS & & & & & & $1(4)$ \\
\hline Dehydration & $1(3)$ & & & $2(7)$ & & \\
\hline Diarrhea & $1(3)$ & & & $3(11)$ & & \\
\hline Dyspnea & $4(13)$ & & & & $1(4)$ & \\
\hline Encephalopathy & & & & $1(4)$ & & \\
\hline Fatigue & $1(3)$ & & & & & \\
\hline Febrile neutropenia & & & & $1(4)$ & & \\
\hline $\begin{array}{l}\text { Generalized muscle } \\
\text { weakness }\end{array}$ & & & & $1(4)$ & & \\
\hline Hyperglycemia & & & & $1(4)$ & & \\
\hline Hypoxia & $1(3)$ & & & $1(4)$ & & \\
\hline Lung infection & $1(3)$ & & & $1(4)$ & & \\
\hline $\begin{array}{l}\text { Lymphocyte count } \\
\text { decreased }\end{array}$ & $1(3)$ & & & $1(4)$ & $1(4)$ & \\
\hline Nausea & & & & $1(4)$ & & \\
\hline Neutrophil count decreased & & & & $1(4)$ & & \\
\hline Platelet count decreased & $1(3)$ & $1(3)$ & & $1(4)$ & & \\
\hline Pneumonitis & $1(3)$ & & & & & $1(4)$ \\
\hline Rash maculopapular & & & & $1(4)$ & & \\
\hline Vomiting & & & & $1(4)$ & & \\
\hline White blood cell decreased & & & & & $1(4)$ & \\
\hline Maximum grade any $\mathrm{AE}$ & $9(30)$ & $1(3)$ & & $6(21)$ & $2(7)$ & $2(7)$ \\
\hline
\end{tabular}

Values are $\mathrm{n}(\%)$.

$\mathrm{AE}$, adverse event; NOS, not otherwise specified; PD-L1, programmed death ligand 1.

unclear in lung cancer. The recently published Lung-MAP S1400I study demonstrated that adding ipilimumab to nivolumab did not improve outcomes compared with nivolumab alone in patients with advanced pre-treated sqNSCLC without prior checkpoint inhibitor therapy. ${ }^{19}$ A recent report of a randomized trial of ipilimumab added to pembrolizumab also showed similar outcomes compared with pembrolizumab alone as first-line therapy in patients with advanced NSCLC and PD-L1 tumor proportion score $\geq 50 \% .{ }^{20}$ In the relapsed setting, a study of patients with advanced NSCLC, predominantly adenocarcinoma, that had failed up to three lines of therapy including PD-(L) 1 checkpoint inhibitors demonstrated an ORR of $5 \%$ in patients with primary or acquired resistance. ${ }^{21}$ In that study, primary resistance was defined as disease progression as the best response within 16 weeks of starting checkpoint inhibition, and acquired resistance was defined as progression after initial complete or partial response or stable disease as the best response to PD-(L) 1 inhibitor therapy. The median duration of response was approximately 6 months (24 weeks).

Studies have identified upregulation of co-inhibitory receptors such as TIM-3 in tumors of patients with advanced lung cancer with acquired resistance to PD-(L) 1 inhibitors as well as decreased antigen presentation and neoantigen loss, and altered metabolism to promote an immunosuppressive tumor microenvironment among other potential mechanisms of resistance. ${ }^{22}$ The presence of mutations in KEAP1, PTEN and other genes have also been associated with checkpoint inhibitor resistance. Current strategies to overcome PD-1 inhibitor resistance include targeting the tumor immune microenvironment via a number of pathways including the vascular endothelial growth factor pathway, beta-catenin, adenosine, and 
others. Novel agents targeting alternate checkpoints such as V-domain immunoglobulin suppressor of T cell activation (VISTA), Lymphocyte-activation gene 3 (LAG-3), $\mathrm{T}$ cell immunoglobulin and mucin domain-containing protein 3 (TIM-3), and T cell immunoreceptor with Ig and ITIM domains (TIGIT) are also in clinical development, as well as other approaches such as cell therapy. A number of combination approaches are also under study through the Lung-MAP Protocol, including the combination of ramucirumab plus pembrolizumab in patients with checkpoint inhibitor refractory disease (S1800A) and an upcoming study of interleukin-15 agonist N-803 with pembrolizumab in this population (S1800D).

In conclusion, the addition of a CTLA- 4 checkpoint inhibitor to ongoing PD-L1 checkpoint inhibitor therapy did not yield meaningful benefit in patients with advanced sqNSCLC and resistance to PD-(L) 1 inhibition, whether primary or acquired. This population of patients should be prioritized for clinical trial enrollment, including through Lung-MAP.

\section{Author affiliations}

${ }^{1}$ Division of Medical Oncology/Hematology, Princess Margaret Hospital Cancer Centre, Toronto, Ontario, Canada

${ }^{2}$ Clinical Research Division, Fred Hutchinson Cancer Research Center, Seattle, Washington, USA

${ }^{3}$ Thoracic Oncology, Columbia University Irving Medical Center, New York, New York, USA

${ }^{4}$ Center for Thoracic Oncology, Tisch Cancer Institute and Icahn School of Medicine Mount Sinai, New York, New York, USA

${ }^{5}$ Department of Radiology, NewYork-Presbyterian/Columbia University Medical Center, New York, New York, USA

${ }^{6}$ Medical Oncology, Heartland NCORP, Decatur, Illinois, USA

${ }^{7}$ Hematology Oncology, Bon Secours Cancer Institute, Richmond, Virginia, USA ${ }^{8}$ Medical Oncology/Hematology, Atlanta Cancer Care Centers, Atlanta, Georgia, USA ${ }^{9}$ Medical Oncology, Duke University Medical Center, Durham, North Carolina, USA ${ }^{10}$ Department of Radiation Oncology, Washington University in St Louis School of Medicine, St Louis, Missouri, USA

${ }^{11}$ Department of Hematology and Medical Oncology, Winship Cancer Institute of Emory University, Atlanta, Georgia, USA

${ }^{12}$ Medical Oncology, Yale Cancer Center I Yale School of Medicine I Smilow Cancer

Hospital at Yale New Haven, New Haven, Connecticut, USA

${ }^{13}$ Department of Thoracic/Head and Neck Medical Oncology, The University of Texas MD Anderson Cancer Center, Houston, Texas, USA

${ }^{14}$ Divison of Hematology and Oncology, Department of Medicine, University of California Davis Comprehensive Cancer Center, Sacramento, California, USA

${ }^{15}$ Division of Hematology/Oncology, Department of Medicine, UC Davis

Comprehensive Cancer Center, Sacramento, California, USA

Contributors NB: conceptualization, investigation, methodology, data curation, validation, project administration, original draft, review and editing; MR: conceptualization, methodology, data curation, formal analysis, software, validation, visualization, review and editing; NR: conceptualization, investigation, methodology, project administration, review and editing; FH: conceptualization, investigation, methodology, review and editing; PM: conceptualization, investigation, methodology, review and editing; LHS: conceptualization, methodology, review and editing; JW: investigation, review and editing; WJI: investigation, review and editing; SCR: investigation, review and editing; JC: investigation, review and editing; JDB: supervision, investigation, review and editing; TS: supervision, investigation, review and editing; SR: supervision, Investigation, review and editing; JM: methodology, data curation, formal analysis; validation, visualization, review and editing; KM: methodology, data curation, formal analysis; validation, visualization, review and editing; RH: conceptualization, investigation, methodology, project administration, review and editing; VAP: conceptualization, investigation, methodology, project administration, review and editing; KK: conceptualization, investigation, methodology, project administration, review and editing; DG: conceptualization, investigation, methodology, project administration, review and editing.

Funding Supported in part by NIH/NCl grants awards U10CA180888, U10CA180819, U10CA180820, U10CA180821, U10CA180863, U10CA180868; and by AstraZeneca, through the Foundation for the National Institutes of Health, in partnership with Friends of Cancer Research.

Disclaimer The content is solely the responsibility of the authors and does not necessarily represent the official views of the National Institutes of Health.

Competing interests The authors' financial disclosures include: NBL received travel support and institutional research funding from Astra Zeneca and personal fees (CME lectures) from MSD and BMS, unrelated to the study. MR salary support from Hope Foundation, participation on DMSB for AstraZeneca, unrelated to study. NR received personal fees from Abbvie, Apricity, AstraZeneca, Boehringer Ingelheim, Calithera, Dracen, Editas, Eli Lilly, EMD Serono, G1 Therapeutics, Genentech, Gilead, GlaxoSmith Kline, Illumina, Merck, Neogenomics, Novartis and Takeda, and personal fees and stock options from Arcus, Belicum, Brooklyn ImmunoTherapeutics, and Gritstone; royalties for patent filed by MSKCC identifying determinants of cancer response to immunotherapy (PCT/US2015/062208 licensed to Personal Genome Diagnostics; PM reports personal fees from Guardant Health. LHS research support from Merck, Bristol-Myers-Squibb, and Boehringer, participating in DSMB for Regeneron, patent with Varian. SCR fees for expert testimony. JC reports consulting fees from Amgen, AstraZeneca, Coherus, Merck, NCCN, Pfizer and travel support from AstraZeneca, GI Therapeutics, participating on DSMB or Ad Board for Beyond Spring, Celegene, G1 Therapeutics, Janssen, Merrimack, Mylan, Roche. JDB is principal investigator of AstraZeneca sponsored trial (PACIFIC 2), research support and consulting fees from Varian. TS institutional grants from Genentech/Roche, Blueprint Medicines, AstraZeneca, Takeda, Advaxis, Regeneron, participation on DSMB or ad board for Takeda, AstraZeneca, Genentech/Roche, Foundation Medicine, Pfizer, EMD Serono, Novartis, Daiichi Sankyo, Lilly, Puma Biotechnology, Janssen Oncology, Regeneron. SR grants from Tesaro, Merck, AstraZeneca, Advaxis, BMS, Amgen, Takeda, Genmab, GSK, consult fees from Amgen, BMS, Genentech/Roche, Merck, AstraZeneca, Takeda, Eisai, Daiichi Sankyo, Sanofi, GSK, Lilly. RSH reports membership in board of directors for Immunocore Holdings Ltd, Junshi Pharmaceuticals, consulting including travel support from AstraZeneca, Genentech/Roche, Merck, Ventana Medical Systems, Inc., consulting with ARMO Biosciences, Bayer Healthcare Pharmaceuticals, Bolt Biotherapeutics, Bristol-Myers Squibb, Candel Therapeutics, Cybrexa Therapeutics, eFFECTOR Therapeutics, Eli Lilly and Company, EMD Serono, Foundation Medicine, Genmab, Gilead, Halozyme Therapeutics, Heat Biologics, I-Mab Biopharma, Immunocore, Infinity Pharmaceuticals, Loxo Oncology, Mirati Therapeutics, Nektar, Neon Therapeutics, NextCure, Novartis, Ocean Biomedical, Oncternal Therapeutics, Pfizer, Ribbon Therapeutics, Sanofi, Seattle Genetics, Shire PLC, Spectrum Pharmaceuticals, STCube Pharmaceuticals, Symphogen, Takeda, Tesaro, Tocagen, Ventana Medical Systems, WindMIL Therapeutics, Xencor. Research support from AstraZeneca, Eli Lilly and Company, Genentech/Roche, Merck. VP research funding from AstraZeneca, BMS, Eli Lilly, Novartis, Merck, F. Hoffman-La Roche, Nektar Therapeutics, Janssen, Bristol-Myers-Squibb, Checkmate, Incyte, consulting fees from AbbVie, Araxes, Arrys Therapeutics, Bolt Therapeutics, Clovis Oncology, Exelixis, G2 Innovation, Gritstone, Ideaya, Leeds Biolabs, Loxo Oncology, Takeda, Tesaro, TRM Oncology, AstraZeneca, BMS, Eli Lilly, Novartis, Merck, F. Hoffman-La Roche, Nektar Therapeutics, Janssen, Bristol-Myers-Squibb, personal fees from F. Hoffman-La Roche, employee of Pfizer. KK travel and consultant fees from AstraZeneca. DRG consultant and participation on advisory boards for AstraZeneca (institutional), Roche-Genentech (institutional), Guardant Health (institutional), 10 Biotech (institutional) and Oncocyte (institutional) fees paid to institution, research funding from Amgen, AstraZeneca, Genentech and Merck, personal fees from Inivata, Lilly, Merck and Novartis. The following investigators have no financial disclosures to report: FH, JW, Wjl, KM, JM.

Patient consent for publication Not required.

Ethics approval The study was conducted in accordance with the Declaration of Helsinki. Study conduct was approved by the ethics review committee of each participating center and all patients provided written informed consent prior to participation.

Provenance and peer review Not commissioned; externally peer reviewed.

Data availability statement Data are available on reasonable request. Data requests may be submitted to SWOG for review (https://www.swog.org/sites/ default/files/docs/2019-12/Policy43_0.pdf)

Supplemental material This content has been supplied by the author(s). It has not been vetted by BMJ Publishing Group Limited (BMJ) and may not have been 
peer-reviewed. Any opinions or recommendations discussed are solely those of the author(s) and are not endorsed by BMJ. BMJ disclaims all liability and responsibility arising from any reliance placed on the content. Where the content includes any translated material, BMJ does not warrant the accuracy and reliability of the translations (including but not limited to local regulations, clinical guidelines, terminology, drug names and drug dosages), and is not responsible for any error and/or omissions arising from translation and adaptation or otherwise.

Open access This is an open access article distributed in accordance with the Creative Commons Attribution Non Commercial (CC BY-NC 4.0) license, which permits others to distribute, remix, adapt, build upon this work non-commercially, and license their derivative works on different terms, provided the original work is properly cited, appropriate credit is given, any changes made indicated, and the use is non-commercial. See http://creativecommons.org/licenses/by-nc/4.0/.

\section{ORCID iDs}

Natasha B Leighl http://orcid.org/0000-0002-3249-4602

Karen Kelly http://orcid.org/0000-0002-2235-6636

David R Gandara http://orcid.org/0000-0003-1784-048X

\section{REFERENCES}

1 Herbst RS, Gandara DR, Hirsch FR, et al. Lung Master Protocol (Lung-MAP)-a biomarker-driven protocol for accelerating development of therapies for squamous cell lung cancer: SWOG S1400. Clin Cancer Res 2015;21:1514-24.

2 Redman MW, Papadimitrakopoulou VA, Minichiello K, et al. Biomarker-driven therapies for previously treated squamous nonsmall-cell lung cancer (Lung-MAP SWOG S1400): a biomarker-driven master protocol. Lancet Oncol 2020;21:1589-601.

3 Brahmer J, Reckamp KL, Baas P, et al. Nivolumab versus docetaxel in advanced squamous-cell non-small-cell lung cancer. N Engl J Med 2015;373:123-35.

4 Borghaei H, Paz-Ares L, Horn L, et al. Nivolumab versus docetaxel in advanced nonsquamous non-small-cell lung cancer. $\mathrm{N}$ Engl J Med 2015;373:1627-39.

5 Herbst RS, Baas P, Kim D-W, et al. Pembrolizumab versus docetaxel for previously treated, PD-L1-positive, advanced non-small-cell lung cancer (KEYNOTE-010): a randomised controlled trial. The Lancet 2016;387:1540-50.

6 Rizvi NA, Hellmann MD, Snyder A, et al. Mutational landscape determines sensitivity to PD-1 blockade in non-small cell lung cancer. Science 2015;348:124-8.

7 Ricciuti B, Recondo G, Spurr LF, et al. Impact of DNA damage response and repair (DDR) gene mutations on efficacy of PD-(L)1 immune checkpoint inhibition in non-small cell lung cancer. Clin Cancer Res 2020;26:4135-42.

8 Mazzotta M, Filetti M, Occhipinti M, et al. Efficacy of immunotherapy in lung cancer with co-occurring mutations in Notch and homologous repair genes. J Immunother Cancer 2020;8:e000946.
9 Curran MA, Montalvo W, Yagita H, et al. Pd-1 and CTLA-4 combination blockade expands infiltrating $T$ cells and reduces regulatory T and myeloid cells within B16 melanoma tumors. Proc Natl Acad Sci U S A 2010;107:4275-80.

10 Motzer RJ, Tannir NM, McDermott DF, et al. Nivolumab plus ipilimumab versus sunitinib in advanced renal-cell carcinoma. $N$ Engl J Med 2018:378:1277-90.

11 Larkin J, Hodi FS, Wolchok JD. Combined nivolumab and ipilimumab or monotherapy in untreated melanoma. N Engl J Med 2015;373:23-34.

12 Drake CG. Combination immunotherapy approaches. Ann Oncol:41-6.

13 Baas P, Scherpereel A, Nowak AK, et al. First-Line nivolumab plus ipilimumab in unresectable malignant pleural mesothelioma (CheckMate 743): a multicentre, randomised, open-label, phase 3 trial. Lancet 2021;397:375-86.

14 Hellmann MD, Paz-Ares L, Bernabe Caro R, et al. Nivolumab plus ipilimumab in advanced non-small-cell lung cancer. N Engl J Med 2019;381:2020-31.

15 Langer CJ, Redman MW, Wade JL, et al. Swog S1400B (NCT02785913), a phase II study of GDC-0032 (Taselisib) for previously treated PI3K-Positive patients with stage IV squamous cell lung cancer (Lung-MAP Sub-Study). J Thorac Oncol 2019;14:1839-46.

16 Edelman MJ, Redman MW, Albain KS, et al. SWOG S1400C (NCT02154490)-A Phase II Study of Palbociclib for Previously Treated Cell Cycle Gene Alteration-Positive Patients with Stage IV Squamous Cell Lung Cancer (Lung-MAP Substudy). J Thorac Oncol 2019;14:1853-9.

17 Aggarwal C, Redman MW, Lara PN, et al. SWOG S1400D (NCT02965378), a Phase II Study of the Fibroblast Growth Factor Receptor Inhibitor AZD4547 in Previously Treated Patients With Fibroblast Growth Factor Pathway-Activated Stage IV Squamous Cell Lung Cancer (Lung-MAP Substudy). J Thorac Oncol 2019;14:1847-52.

18 Antonia S, Goldberg SB, Balmanoukian A, et al. Safety and antitumour activity of durvalumab plus tremelimumab in non-small cell lung cancer: a multicentre, phase 1B study. Lancet Oncol 2016;17:299-308.

19 Gettinger SN, Redman MW, Bazhenova L. A phase III study of nivolumab plus ipilimumab versus nivolumab for previously treated patients with stage IV squamous cell lung cancer (Lung-MAP SubStudy S1400I, NCT02785952. Manuscript submitted for publication.

20 Boyer M, Şendur MAN, Rodríguez-Abreu D, et al. Pembrolizumab plus ipilimumab or placebo for metastatic non-small-cell lung cancer with PD-L1 tumor proportion score $\geq 50 \%$ : randomized, double-blind phase III KEYNOTE-598 study. J Clin Oncol 2021;39:2327-38.

21 Garon EB, Spira Al, Goldberg SB, et al. Safety and activity of durvalumab + tremelimumab in immunotherapy (IMT)-pretreated advanced NSCLC patients. JCO 2018;36:9041.

22 Koyama S, Akbay EA, Li YY, et al. Adaptive resistance to therapeutic $\mathrm{PD}-1$ blockade is associated with upregulation of alternative immune checkpoints. Nat Commun 2016;7:10501. 